\title{
Augmenting Mathematics Courses by Problem-Based Learning
}

\author{
http://dx.doi.org/10.3991/ijep.v6i1.5368 \\ M. Frank and C. Roeckerath \\ RWTH Aachen University, Aachen, Germany
}

\begin{abstract}
We describe a project that aims to motivate undergraduate engineering students to participate more in mathematics courses. Our approach shares characteristics of active, project-based and problem-based learning. We have developed interactive projects that deal with the mathematics behind real-world applications. In this paper, we describe one example project in detail, and discuss our guiding principles in designing projects. The projects are open in the sense that there are many possible solutions, and several possible follow-up questions. We use a MATLAB environment, which helps intuitive understanding of mathematical notions, lowers the barrier to programming, and provides many interfaces. Our activities are bundled in an education lab, which we also briefly describe. We conclude with an assessment of the impact of the supplementary courses.
\end{abstract}

Index Terms-Digital and online engineering education, Open and project based learning, Sustainable energy for all

\section{INTRODUCTION}

"Why do I need to study mathematics? I just want to build cars."

"Mathematics is solving problems with pen and paper. Programming a computer is computer science."

Contrary to engineering, mathematics as a science is method-oriented and not problem-oriented. In undergraduate courses as well as in high-school classes, a theoretical structure is built from the ground up, and the main purpose is to introduce notions in a consistent and systematic way. For instance, one typically introduces the natural numbers, then integers, followed by the rational numbers, real numbers, and finally complex numbers. This is subsequently extended to vector spaces. While this way of teaching certainly is reasonable, it leads to motivation problems among the participants. "Why do I need to study this?" is a frequently asked question. Often, students recognize the usefulness of mathematics too late in their studies.

Many initiatives aim at changing the classical university and especially engineering education. One of the reasons is to motivate students. Also, it has been recognized that the landscape of engineering in society has dramatically changed. Engineers (and scientists) are not only problem solvers anymore, but also problem definers, and members of multidisciplinary, diverse, international teams. It has therefore been argued that there is a need for new learning styles that foster creativity and innovative thinking. Examples of these initiatives are CDIO (Conceive-DesigImplement-Operate) [6], ICTMA (International Study Group for the Teaching of Mathematical Modelling and Applications, cf. the book series [7]), or Computer-Based

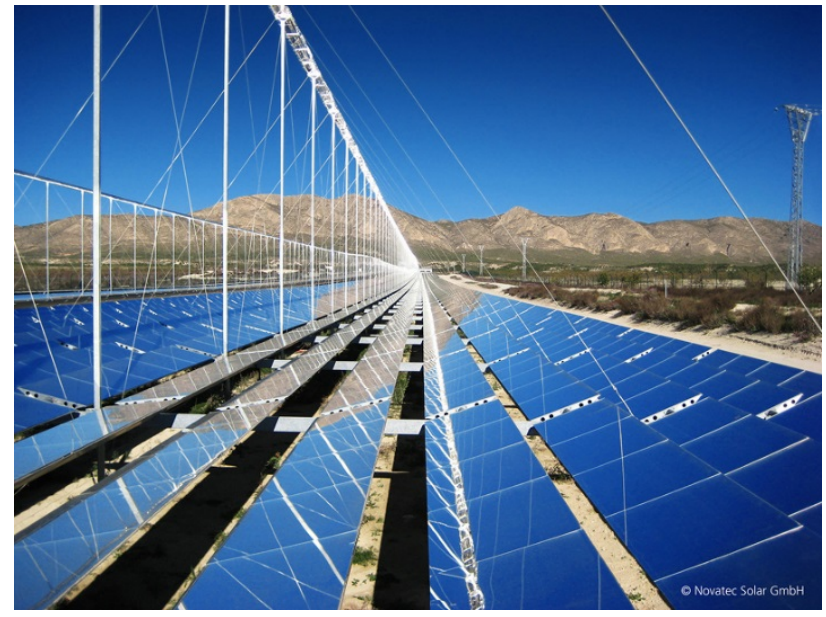

Figure 1. Field of mirrors in a Fresnel plant (source: Novatec Solar $\mathrm{GmbH}$ ). What are optimal mirror sizes and positions? How high does the pipe have to be?

Math [9], and the many active and problem-based learning initiatives [10].

We try to contribute to this growing field by developing interactive, problem-oriented material that accompanies the transition period from high-school into university, i.e. the final years of high-school and the first years of undergraduate studies (cf. Figure 2). We specifically do not modify the method-oriented structure of the courses themselves, because we believe that method-oriented thinking is useful for students. Undoubtedly, questioning and improving the available tools (which we understand as method-oriented thinking) often leads to advances in engineering designs. Mathematics is often the only strict method-oriented course in an engineering curriculum.

In the projects, on the other hand, mathematics is introduced in a playful way, which is directly aimed at the problem solution. We take a practical technology from everyday life and show the mathematics behind it. The mathematical notions that are new to the students are introduced exactly at the point when they are needed. The material is supported by simple MATLAB programs. In that way students can easily experiment with different model refinements and mathematical solution techniques. Our goal is to spark interest for higher mathematics by demonstrating the practical relevance of abstract notions, to motivate, and to provide students with positive feedback.

Our approach has some aspects of project-based [5] and problem-based learning [1]. According to Blumenfeld and Krajcik [5] there are several studies that demonstrate the positive effects of project-based learning concerning the 


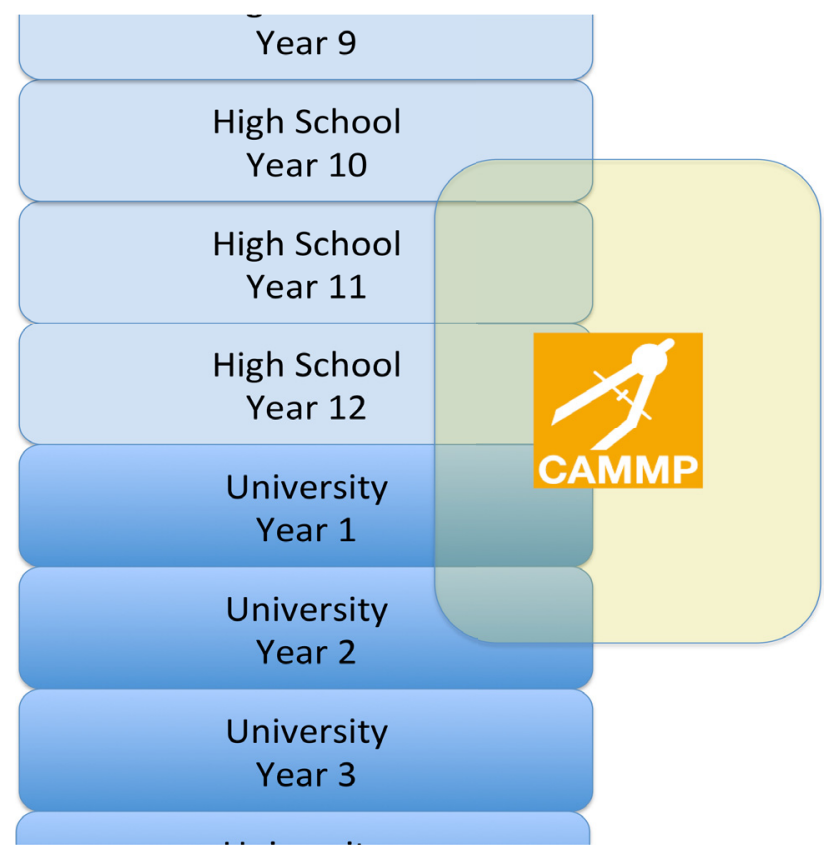

Figure 2. CAMMP adresses students of the final years of high school as well as first-and second-year undergraduates.

learning effect of students. Many other projects, papers and initiatives exist. However, because of the vastness of the literature and limited space, our list must be incomplete. A problem-based concept to augment mathematical education in mechanical engineering was realized in the winter term 2012/13 at the University of Applied Sciences Kiel. The students that attended to this course also aimed a better learning effect than the students of the regular courses [2]. Another possibility to augment mathematical education for undergraduate or high school students are mathematical modeling weeks where real world problems have to be solved with mathematics. Working on these problems similar to problem-based learning, new mathematical concepts and methods are discovered when they are needed to solve the real world problem. In this way students learn the fundamental meaning of mathematics for other sciences, industry and every day life. In the framework of modeling weeks students deal with modeling problems which are authentic and do not have an optimal or unique solution. In [4], a classification of the recent modeling approaches is given. According to this classification our described modeling activities correspond to the realistic or applied modeling perspective. In Germany there are a few modeling weeks such as our concept and the modeling week of TU Kaiserslautern [3].

This paper is an extended and updated version of a conference contribution [15]. Its remainder is organized as follows. In the following section we describe an example and how we have implemented it with interactive MATLAB codes. Our design principles are described and put into context in Section III. In Section IV, we describe the organizational framework of the education lab CAMMP at RWTH, before concluding with an outlook.

\section{AN EXAMPLE}

We try to explain our approach with the example project Optimizing a solar power plant, which we have used several times with undergraduate and even high school students.

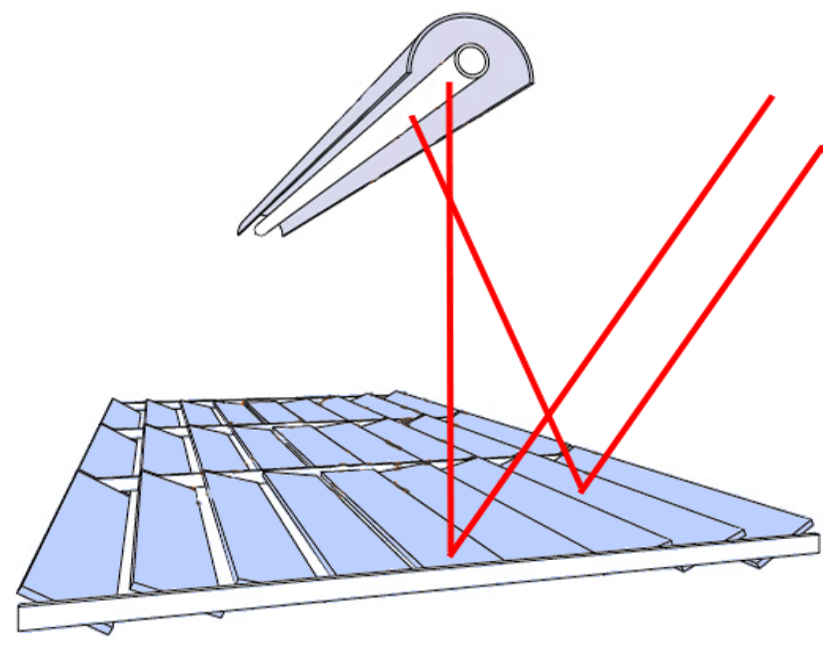

Figure 3. Secondary reflector light path. Light from the sun is reflected by plane mirrors, and then hits the absorber tube either directly, or is captured by a secondary reflector, and then bundled onto the absorber tube.

The energy sector is currently in a transition towards renewable energies. Solar thermal power plants are one possibility for sustainable energy production. When constructing such power plants, one searches for the most economical design. This is a setup, which maximizes energy production at minimal costs.

A Fresnel solar thermal power plant consists of long plane mirrors that concentrate sunlight onto absorber tubes $(0)$, and thus heats the liquid contained in these tubes. Via a heat exchanger, this hot liquid is used to evaporate water, which in turn drives a turbine. A secondary reflector around the absorber pipe collects secondary radiation that would otherwise miss the pipe (Figure 3. ).

In a workshop that can last from 90 minutes to a whole day, students develop a mathematical model to describe the energy production of such a power plant. They use real sun data from a known plant site. The main tool is a MATLAB code, in which the students fill-in missing lines and formulas (Figure 6. ). At the same time, the code provides feedback by displaying the result graphically (Figure 7. ). This entices the students to re-think and refine their formulas.

The first question is: Depending on the sun position, into which direction does a mirror have to point such that the sunlight is reflected onto the absorber? We make the following model assumptions. All considerations will take place in 2D (Figure 4. ), while an extension to 3D can easily be accomplished. Furthermore we assume that the sunrays are parallel (a sun band) and that all radiation captured by the secondary reflector reaches the absorber pipe. In the beginning we only consider a single mirror which is positioned directly under the absorber tube.

In the first task students need to determine $\gamma$, the angle of inclination for the mirror, which is the angle between the horizon and the normal of the mirror. The angle between the incident sunrays and the horizon is $\alpha$. Since in this setup the mirror is positioned exactly below the pipe, the angle $\beta$ between the horizon and the reflected sunrays is $\frac{\pi}{2}$. Thus the angle of inclination $\gamma$ must be determined by the angle of the sun incidence $\alpha$. Using the law of reflec- 


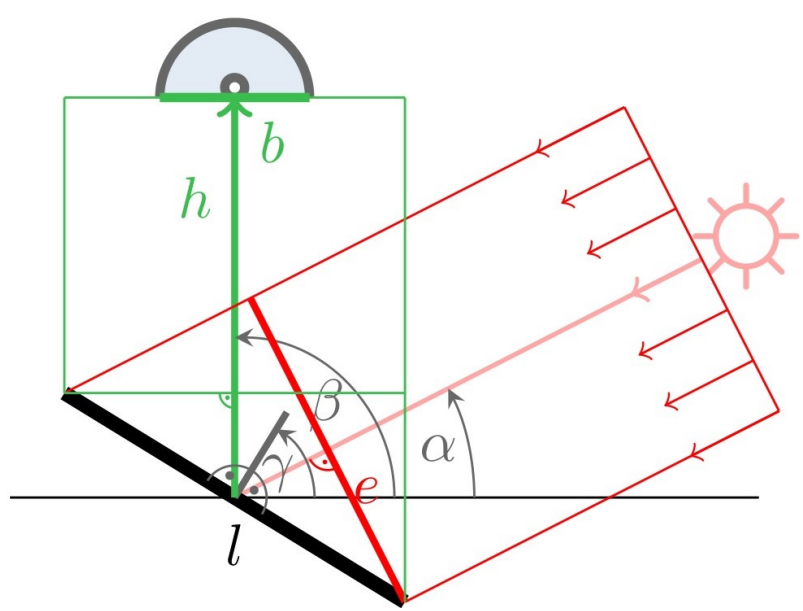

Figure 4. The geometry of mirror positioning with incident radiation (red) and reflected radiation (green): $\alpha$ angle of the sun over the horizon, $\beta$ angle between the horizon and the reflected sun rays (in this setup $\beta=90^{\circ}$, since the mirror is positioned exactly below the pipe), $\gamma$ mirror angle so that the sun rays are reflected into the direction of the absorber tube, $l$ width of the mirror, $h$ the height of the absorber tube, $b$ width of the secondary reflector, incident radiation band $e$.

tion, which states that the angle of incidence is equal to the angle of reflection, and simple angle theorems it is immediately clear, that $\beta-\gamma=\gamma-\alpha$ and thus

$$
\gamma=\frac{1}{2}(\alpha+\beta)=\frac{1}{2}\left(\alpha+\frac{\pi}{2}\right)
$$

However, the students are quite free in the triangles and the sine and cosine theorems they use.

Since in the first task the mirror was put in the correct position the second task is to compute the amount of power $P_{M}$ reaching the mirror. After that the ratio $a$ of the reflected radiation captured by the secondary reflector to the total incoming radiation can be determined. Using these two results one can calculate the power $P_{R}$ reaching the absorber tube. Let the width of the mirror be $l$. The irradiation density is given in power per meter. One can refer to the width of the reflected radiation band $e$ as the effective mirror size. Using some theorems about angles and the angle sum in a triangle the angle between the mirror and the sun radiation band can be determined as $\gamma-\alpha$. Thus the effective mirror size is $e=l \cdot \cos (\gamma-$ $\alpha)$. Therefore we get $P_{M}=e \cdot L$ for the power reaching the mirror.

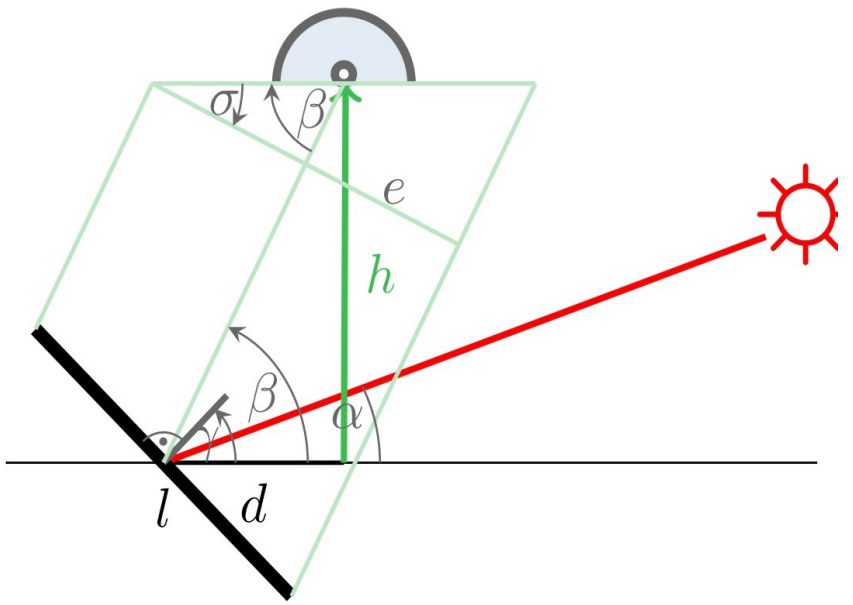

Figure 5. Model of a shifted mirror. Incident sun ray (red); reflected sun band (green); mirror shift d.
Also the reflected sun band has the width e and thus the power $P_{M}$. Since the sunrays incide perpendicularly on the secondary reflector, the ratio $a$ of the reflected sun band, which reaches the secondary reflector and therefore the absorber tube, is $a=\min \{1, b / e\}$. Finally, the power $P_{R}$ reaching the absorber tube can be determined by

$$
P_{R}=a \cdot P_{M} \text {. }
$$

While at first the mirror is directly located under the pipe, in the third task it is moved to the side by the distance $d$ (Figure 5. ).

The height of the absorber tube is $h$. One gets

$\beta= \begin{cases}\arctan \frac{h}{d}, & d<0 \\ \pi / 2, & d=0 \\ \pi-\arctan \frac{h}{d}, & d>0\end{cases}$

by using the tangent.

Also in case of the shifted mirror the angle of inclination $\gamma$ can be determined by $\gamma=\frac{1}{2}(\alpha+\beta)$. And also the power reaching the absorber tube can be computed in three steps: The calculation of the amount of power reaching the mirror does not change: $P_{M}=e \cdot L$ with

$$
e=l \cdot \cos (\gamma-\alpha) \text {. }
$$

The ratio $a$ of the reflected sun band, which reaches the secondary reflector, is $a=\min \{1, b \cdot \cos \sigma / e\}$ with $\sigma=|\beta-\pi / 2|$. As before, the power $P_{R}$ reaching the absorber tube can be determined by

$P_{R}=a \cdot P_{M}$.

Once students are able to calculate the produced power for any sun position, real sun data is used to compute the energy production per year. This can be solved by numerical integration. Students very easily develop some kind of rectangle or trapezoidal rule, two well-known quadrature methods, by themselves without much help. As a next step the students have to refine the model by considering disturbances like the following: Sunrays are not really parallel, the mirror surfaces are not optimal flat and a perfect alignment of the mirrors is not practicable.

Then a simple optimal design can be computed: Given a fixed mirror size and pipe/secondary reflector size, how high does the pipe need to be above the ground? If a pipe is too low, not enough radiation will reach it. If on the

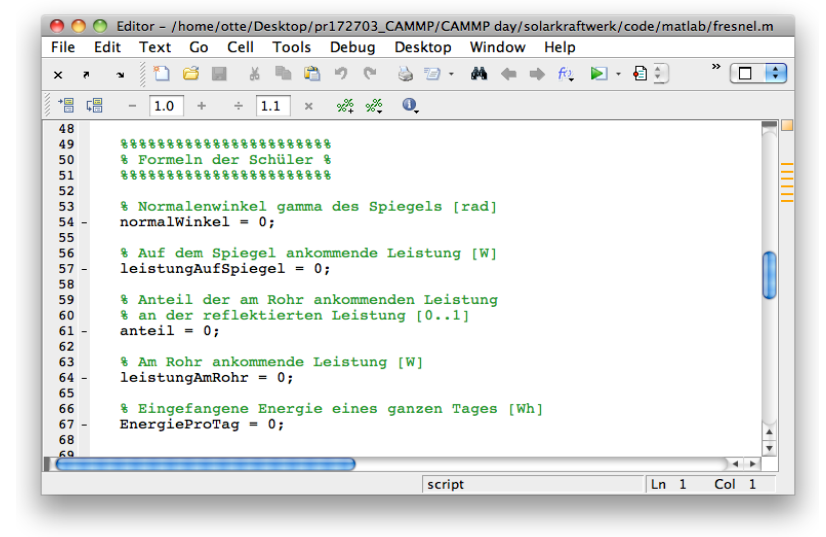

Figure 6. Sample MATLAB code (in German). In line 54, students have to enter a formula for the angle $\gamma$ from the previous figure, in terms of the sun angle. Line 57 requires the computation of the effective mirror size and thereby the sun power on the mirror. Similar formulas have to be entered in lines 61, 64 and 67 . 
other hand it is too high, the atmospheric attenuation will prevent radiation from reaching it. Thus there must be an optimal value which students can compute either by trialand-error, or by simple optimization techniques.

The problem can even be extended, so that students pass several times through the modeling cycle (Figure 8). Starting from the real situation, a simplified situation is defined (e.g. working in 2D). A mathematical description (e.g. using trigonometry) is followed by a numerical solution and interpretation of the results. Beside the trigonometric solution explained above the problem allows the application of a wide range of different mathematical techniques. One can add multiple mirrors and thus blocking and shading. This can again be solved by trigonometry; the solution is more complicated though. One can add curved mirror surfaces (which is actually done in reality). Then trigonometry will not work anymore. However, a ray-tracing algorithm will, and is a solution that many students find on their own. The optimization problem can also ask for several more optimal parameters, like e.g. mirror size, distance between mirrors or size of the secondary reflector (which also blocks the sun and thus should not be too big). The optimal design of the secondary reflector is another interesting project. It can be modeled as the solution to an ordinary differential equation! Sensitivity analysis is another natural topic to tackle: How sensitive are the optimal parameters to weather changes?

\section{METHODS}

The methods we employ are centered around several guiding principles by which we choose our problems, and the tools we employ. In each subsection, we have mentioned similar approaches that we are aware of and suggestions for further reading. We are certain that the list is far from complete.

\section{A. All projects are real}

Many textbooks in school use pseudo-applications in the sense that an application is artificially built around an otherwise standard task. For example:

The height of a rollercoaster is given by the function $\mathrm{f}(\mathrm{x})=\left(\mathrm{x}^{2}+2 \mathrm{x}-4\right) \mathrm{e}^{\mathrm{x}}$. Find the maximal height of the rollercoaster.

Students of course realize that they are not really constructing a rollercoaster. Questions like the one above actually have an adversarial effect: Students tend to believe less in the relevance of mathematics for everyday life.

Our problems, on the other hand, are real. The solar power plant problem actually is a simplified version of a past $\mathrm{PhD}$ project. When the project is finished students can compare their results with the parameter values that are used in actual solar power plants. These parameters agree very well with the ones obtained by the very simple model that was developed in the workshop.

\section{B. Mathematics solves real-world problems}

Our aim is that students see a connection to the undergraduate course they are taking. In our case, we combine the project on solar power plant optimization either with the first-year course on linear algebra and geometry, or the second-year course on optimization methods. Our other projects also fit certain topics: We offer a course on

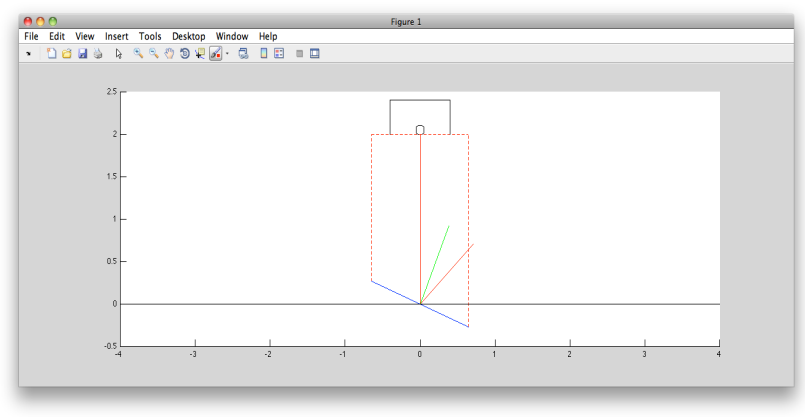

Figure 7. A simple graphical display provides immediate feedback. Shown are sun angle, mirror angle, and the sun image on the absorber.

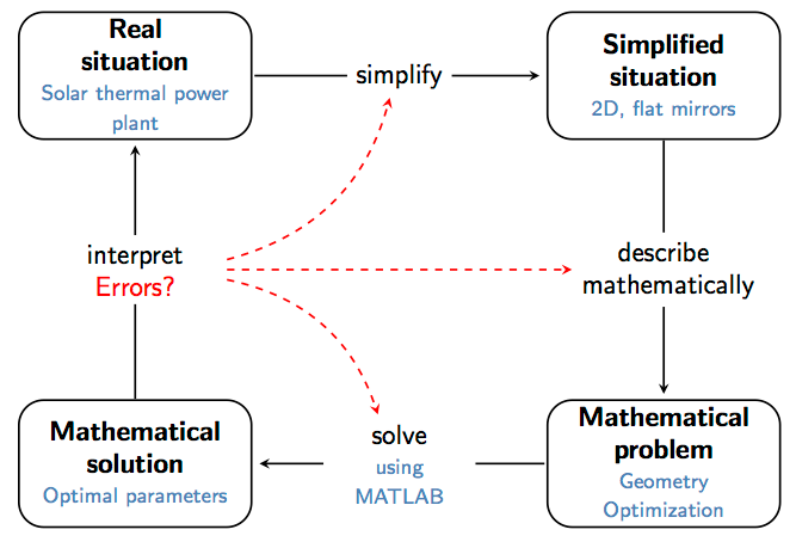

Figure 8. The modeling cycle in our project.

stability of bridges, which is an application of linear systems of equations. Our course on GPS needs curved coordinate systems and nonlinear least squares methods, the technologies MP3 and JPEG use the Fourier transform, possibly in its incarnation as Fast Fourier Transform (FFT).

For high-school students we try to have as few prerequisites as possible. They acquire the necessary tools on the spot, like sine and cosine identities in triangles. Still, the significance of mathematical models for the real world becomes apparent.

\section{Everyone can think in algorithms}

We have observed that many students shy away from writing even simple computer codes. This is in spite of the impression that they can very well think in terms of algorithms. We believe that this is due to unnecessary hurdles that many programming languages put into one's way. One has to declare variables, write header files, use object classes, and so on. To overcome this problem, we use MATLAB. One can directly enter mathematical expressions, without declaring the types in advance. Vectors and matrices are the basic types, and can be used very intuitively. Visualization of a result is extremely simple. There exist many built-in functions, including linear algebra solvers and optimization routines.

Interfaces to e.g. webcam and microphones exist, which we use for the projects on image and music compression. It is possible to read many formats, e.g. GPS raw data.

The idea to use MATLAB came when we observed to students who came up with an algorithm, but did not dare to touch a computer. A group advisor put them in front of MATLAB, and told them to write a code or pseudo-code 
as well they could. He then corrected some very few mistakes, and the program was running.

We encourage students to write code together on a laptop that is connected to a projector. This involves more people in the group. We believe it is essential that engineers view computers as a design tool. This tool becomes ever more important. Our hope is that they do not use it as a black box though, but be curious as to what is inside.

Viewing algorithms as the new literacy in our digitized world is an idea that has been put forward by many authors recently $[11,12]$.

\section{There is more than one solution}

Many mathematics textbook problems, especially proofs, are like jigsaw puzzles: Given are some ingredients (assumptions), prove the following claim. It is pretty clear to students that the problem is designed so that they need to use all assumptions. However, they do not learn to come up with hypotheses. Furthermore, creativity is very limited by the several assumptions that need to be pieced together. Open problems as an alternative way of teaching mathematics have been put forward frequently $[13,14]$.

Our problems are open in the sense that there is more than one way to solve a problem. For instance, the calculation of the mirror direction can be done by very different trigonometric theorems. It can also be solved by linear algebra: The sunrays are described as parallel lines, the mirror is a line too. Sunrays are reflected on that line, which can e.g. be written down as a matrix vector operation. One can consider forward (follow the ray from the sun to the pipe) and backward rays (start at the pipe and see if the ray came from the sun). Our code that provides feedback only checks if the formula gives the correct result, and displays the students' result.

\section{E. Science is never finished}

We also want to stay clear of a too narrow view of a solution. Students should learn that a design process is never finished. During the process, often times more questions appear than were successfully addressed. In the solar power plant example we ask just this in the additional tasks: What about more than one mirror? What if the mirror is curved? Should the secondary receiver be shaped like a parabola, or is another shape optimal? The students have to focus on one aspect in order to finish with a result in the given timeframe. However, we hope that they become aware of other potential challenges. This, again, is different from a typical exercise, which has a clear question and a clear answer, and is done.

\section{ORGANIZATIONAL FRAMEWORK}

So far we have implemented a total of five projects (Stability of bridges, How does Google work?, Optimization of a solar power plant, How does GPS work?, How do MP3 and JPEG work?), with several to follow. The projects are regularly used in the Bachelor course Computational Engineering Science at RWTH Aachen, Germany.

The projects were designed within the Education Lab CAMMP (Computational and Mathematical Modeling Program). CAMMP is a teaching and learning laboratory of mathematical modeling at RWTH, which addresses students of the final high school years as well as first-and second-year undergraduates, cf. Figure 2. . CAMMP aims at pointing out the social relevance of mathematics and simulation sciences. Depending on the constraints like working time, group size and amount of advisors we try to find an optimal balance between active and passive as well as method-oriented and problem-based learning.

CAMMP brings together undergraduate as well as high school students with university researchers to introduce the basics of computational modeling through hands-on exploration. Under the supervision of faculty and researchers, students construct a solution to a challenging real-world problem drawn from industry, business or government. The solution will require the use of both analytical tools as well as numerical simulations. Students will also expand their team-building skills. Moreover, CAMMP gives students an insight into professional careers in science, technology, engineering, and mathematics.

Beside one-day workshops, CAMMP also offers modeling weeks. In that format, a group of typically six students works for one week on a real-world problem. One academic advisor who gives as little hints as possible guides the group. The group develops computer code, prepares a report, and gives a presentation at the end of the week. Example projects are: How can one measure velocities of cars with a cell phone camera? or How can one optimize the booking system of a car sharing company? Again, the projects are real; company representatives attend the final presentation. Each student is encouraged to write code.

\section{OUTCOMES \& CONCLUSIONS}

We have observed that the attendance (which is not mandatory at our university) in project courses is higher than in the normal exercise sessions. Furthermore, students are more motivated to do exercises after the projects. In a few cases, students have continued to work on the additional questions of the projects by themselves. The projects receive very good marks in teaching evaluations.

During our modeling weeks, students regularly work at night on their own. Having a problem to crack and a limited timeframe appears to be very motivating

We believe that augmenting mathematics courses by project-based learning modules can be very beneficial for the motivation of the students. It leads to increased participation, and finally also to higher success.

The materials are available from the authors (currently only in German).

\section{REFERENCES}

[1] H.S. Barrows, "Problem-Based Learning in Medicine and Beyond: A Brief Overview," in Bringing Problem-Based Learning to Higher Education: Theory and Practice, L. Wilkerson, W. H. Gijselaers, Eds., San Franscisco: Jossey-Bass, 1996, pp. 3-12. http://dx.doi.org/10.1002/t1.37219966804

[2] S. Beinhauer, D. Krüger, A. Nessel and S. Schmidt, "Problembasiertes Lernen in der Mathematik, " in Mathematik im Übergang Schule/Hochschule und im ersten Studienjahr, A. Hoppenbrock, S. Schreiber, R. Göller, R. Biehler, B. Büchler, R. Hochmuth, Eds. in press.

[3] M. Bracke, S. Göttlich and T. Götz, "Modellierungsproblem Dart spielen“, in Mathematisches Modellieren für Schule und Hochschule - Theoretische und didaktische Hintergründe, R. Borromeo Ferri, G. Greefrath and G. Kaiser, Eds. Wiesbaden: Springer Spektrum, 2013, pp. 147-162. http://dx.doi.org/10.1007/978-3658-01580-0 7

[4] G. Kaiser, B. Schwarz, "Authentic Modelling Problems in Mathematics Education-Examples and Experiences," Journal für 
PAPER

Augmenting Mathematics Courses by Problem-BASed LeARning

Mathematik-Didaktik, $\quad 31(1), \quad 2010, \quad$ pp. $\quad 51-76$. http://dx.doi.org/10.1007/s13138-010-0001-3

[5] P. Blumenfeld, S. Krajcik "Project-Based Learning," in R. K. Sawyer, The Cambridge Handbook of the Learning Sciences, New York: Cambridge University Press, 2006, pp. 317-334.

[6] Edward Crawley; Johan Malmqvist; Sören Östlund; Doris Brodeur: "Rethinking Engineering Education, The CDIO Approach," Springer, 2007, ISBN 978-0-387-38287-6.

[7] G. Kaiser, G.A. Stillman: "International Perspectives on the Teaching and Learning of Mathematical Modelling", book series.

[8] M. Prince: "Does active learning work? A review of the research," J. Engr. Education 93, 2004, pp. 223-231. http://dx.doi.org/10.1002/j.2168-9830.2004.tb00809.x

[9] Computer-based

Mathematics, http://www.computerbasedmath.org

[10] R.B. Barr, J. Tagg: "From Teaching to Learning - A New Paradigm for Undergraduate Education," Change: The Magazine of Higher Learning 27, 1995, pp. 12-26 http://dx.doi.org/10.1080/00091383.1995.10544672

[11] M. Csernocj, P. Biró, "The power in digital literacy and algorithmic skill,“ Procedia - Social and Behavioral Sciences 174, 2015, pp. 550-559. http://dx.doi.org/10.1016/j.sbspro.2015.01.705

[12] A. Tarchini, "Why creating algorithms is the 'new literacy", conference presentation at World Engineering Education Forum (WEEF) Florence, 2015.
[13] $\mathrm{H} . \mathrm{Wu}$, "The role of open-ended problems in mathematics education“, J. of Math. Behavior 13, 1994, pp. 115-128. http://dx.doi.org/10.1016/0732-3123(94)90044-2

[14] E. Pehkonen, "Open problems as means for promoting mathematical thinking and understanding," In: András Ambrus, Éva Vásárhelyi (Eds.), Problem Solving in Mathematics Education. Proceedings of the 15th ProMath conference, 2015, pp. 152-162.

[15] M. Frank, M. Hattebuhr, C. Roeckerath, "Augmenting Mathematics Courses by Project-Based Learning," Proceedings of 2015 International Conference on Interactive Collaborative Learning, 2015 .

\section{AUTHORS}

M. Frank is with the Department of Mathematics (MATHCCES), RWTH Aachen University, Aachen, Germany (e-mail: frank@mathces.rwth-aachen.de).

C. Roeckerath is with the Department of Mathematics (MATHCCES), RWTH Aachen University, Aachen, Germany (e-mail: roeckerath@mathces.rwth-aachen.de).

This article is an extended and modified version of a paper presented at the International Conference on Interactive Collaborative Learning (ICL2015), held 20-24 December 2015, in Florence, Italy. It was supported in part by the German Federal Ministry for Education and Research through the program "Qualtätsoffensive Lehrerbildung. Submitted 15 December 2015 . Published as resubmitted by the authors 28 January 2016. 